\title{
Incidencia de infección por SARS-CoV-2 en tres clínicas de fertilidad en México
}

\section{Incidence of SARS-CoV-2 infection in three fertility clinics in Mexico}

\author{
Miguel Ángel Ulloa Miranda, * Gabriela García Jiménez, ${ }^{\ddagger}$ \\ Salvador Espino y Sosa, ${ }^{\ddagger}$ Sandra Rojas Peñaloza, ${ }^{*}$ Citlali Lorenzo Bautista, ${ }^{*}$ \\ Lizbeth Martínez Portugal,* Lucía Escobedo Berumen ${ }^{\S}$
}

Citar como: Ulloa MMÁ, García JG, Espino y Sosa S, Rojas PS, Lorenzo BC, Martínez PL, Escobedo BL. Incidencia de infección por SARS-CoV-2 en tres clínicas de fertilidad en México. Acta Med GA. 2021; 19 (4): 514-518. https://dx.doi.org/10.35366/102538

\section{Resumen}

Introducción: Durante la pandemia de COVID-19 se ha venido estudiando de manera paulatina la afectación del virus en las pacientes embarazadas, lo cual provocó un retraso en el deseo de lograr una concepción. Objetivos: Describir la incidencia de infección por SARS-CoV-2 en centros de fertilidad en México. Material y métodos: Es un estudio descriptivo, transversal, observacional, retrospectivo en tres clínicas de reproducción asistida en la Ciudad de México. Se incluyeron mujeres ovodonadoras y candidatas a procedimientos de reproducción asistida con prueba de PCR para SARS-CoV-2. Resultados: Se incluyeron 50 pacientes, 84\% con infertilidad y $16 \%$ ovodonadoras con una media de edad de $35.4 \pm 6.25$ años. De las pacientes, 55\% tenían edad materna avanzada, $59.5 \%$ infertilidad primaria y $40.5 \%$ secundaria, predominando como comorbilidad hipotiroidismo. Se encontraron cuatro pacientes con PCR positiva para SARS-CoV-2 $(p=0.041)$, tres en contacto con personas positivas $(p=0.003)$ y con síntomas en los últimos 15 días $(p=0.371)$. Conclusiones: La incidencia de infección por SARS-CoV-2 en los centros de fertilidad es significativamente baja. No hay que retrasar los procedimientos de reproducción asistida, deben procurarse siempre las máximas medidas de protección sanitaria tanto a las pacientes como al personal de los centros de fertilidad.

Palabras clave: Infertilidad, SARS-CoV-2, concepción.

\section{Abstract}

Introduction: During the COVID-19 pandemic, the impact of the virus in pregnant patients was gradually studied, which caused a delay in the desire of some mothers to achieve conception. Objectives: To describe the incidence of SARSCoV-2 virus infection in fertility centers in Mexico City. Material and methods: It is a descriptive, cross-sectional, observational, retrospective study, carried out in three assisted reproduction clinics in Mexico City. Egg donor women and candidates for assisted reproduction procedures who underwent the SARSCoV-2 PCR test were included. Results: A sample of 50 patients was obtained, $84 \%$ with infertility and $16 \%$ ovodonators with a mean age of $35.4 \pm 6.25$ years. $55 \%$ with advanced maternal age, $59.5 \%$ with primary infertility, and $40.5 \%$ secondary, predominating as comorbidity hypothyroidism. Four patients were found with positive CRP for SARS-CoV-2 ( $p=$ $0.041)$, three in contact with positive people $(p=0.003)$ and with symptoms in the last 15 days $(p=0.371)$. Conclusions: The incidence of SARS-CoV-2 infection in fertility centers is significantly low. Assisted reproduction procedures should not be delayed, always seeking the maximum health protection measures, towards patients and the staff of fertility centers.

Keywords: Infertility, SARS-CoV-2, conception.
* Ginecología y Obstetricia, Residente de Alta Especialidad de Infertilidad y Reproducción Asistida de la Asociación Mexicana de Medicina de la Reproducción. México.

* Ginecología y Obstetricia, Profesor del Curso Alta Especialidad de Infertilidad y Reproducción Asistida de la Asociación Mexicana de Medicina de la Reproducción. México.

$\S$ Maestra en Ciencias Médicas, Gastroenterología y Nutrición Pediátrica del Instituto Nacional de Pediatría. México.

\section{Correspondencia:}

Dr. Miguel Ángel Ulloa Miranda

Correo electrónico: doctorulloamiranda@gmail.com

Aceptado: 29-07-2021.

www.medigraphic.com/actamedica

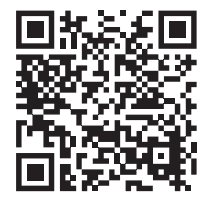




\section{INTRODUCCIÓN}

En 2019 se identificó un nuevo coronavirus como la causa de casos de neumonía en Wuhan, una ciudad en la provincia de Hubei de China. Se propagó rápidamente, dando como resultado una epidemia. ${ }^{1}$ En febrero de 2020 la Organización Mundial de la Salud designó a la enfermedad como COVID-19, el virus que la causa se denomina coronavirus 2 del síndrome respiratorio agudo severo (SARS-CoV-2). ${ }^{2}$ El virus tiene un periodo de incubación estimado de 14 días posteriores a la exposición, y la mayoría de los casos ocurren aproximadamente de cuatro a cinco días después de la exposición (mediana de cuatro días, con rango de dos a siete días). ${ }^{2}$

Acorde a datos de casos confirmados y divulgados públicamente en China con exposición identificable, un estudio de modelado estimó que los síntomas se desarrollarían en $2.5 \%$ de las personas infectadas en 2.2 días y en $97.5 \%$ de las personas infectadas en 11.5 días. La mediana del periodo de incubación en este estudio fue de 5.1 días. ${ }^{3}$

Con respecto a la infertilidad y las infecciones por el agente SARS-CoV-2 surgieron dos interrogantes, la primera en relación a si existe o no una infección vertical; la evidencia hasta el momento revela que no hay transmisión vertical de SARS-CoV-2. La segunda interrogante cuestiona la posibilidad de infección de los gametos en las técnicas avanzadas de reproducción asistida; sin embargo, la Sociedad Europea para la Reproducción y Embriología Humana (ESHRE) sugirió evitar el inicio de nuevos embarazos a través de la transferencia de embriones por ciclos diferidos, pero la razón no está fundamentada en la infección de gametos, sino en evitar que los pacientes viajen para recibir tratamiento de fertilidad y prevenir el estrés adicional en los sistemas de salud. ${ }^{4}$ Albertini menciona que la fecundidad es lo primero que sufre cuando un organismo vivo sufre desafíos de esta magnitud para la salud. ${ }^{5}$

Aunque se habla de una asociación entre las infecciones virales e infertilidad, hasta el momento no hay informes en la literatura sobre el impacto de las infecciones por SARS-CoV-2 en la fertilidad humana. Xu y colaboradores describieron seis casos de orquitis en autopsias de hombres que murieron por SARS-CoV-2 en 2006. Xu y su equipo advirtieron sobre un posible impacto negativo en la fertilidad de los sobrevivientes del SARS-CoV. El grupo de Fan y colaboradores reforzaron la alta expresión de ACE2 en los testículos (conductos seminíferos y células de Leydig), destacando la posibilidad de reducción de la fertilidad masculina en casos de COVID-19. ${ }^{6}$

Aunque se sugiere programar la criopreservación de ovocitos y de embriones para poco después de que haya pasado el pico de infección por COVID-19 en cada país, ${ }^{7}$ no es algo que pueda postergarse por mucho tiempo más, puesto que es una necesidad social poder ofrecer embarazos obtenidos por técnicas de reproducción asistida. Toda vez que a nivel mundial los bebés obtenidos por estos medios representan aproximadamente $0.3 \%$ de la tasa total de nacimientos vivos cada año, se sabe que se realizan más de 1,500,000 ciclos de fertilización in vitro cada año en todo el mundo, lo que resulta en alrededor de 400,000 bebés nacidos. ${ }^{7,8}$

Hay que considerar que en términos de fertilidad la edad de los ovocitos es un factor esencial, por lo que el esperar meses o años antes del tratamiento puede cambiar radicalmente el pronóstico reproductivo de ciertas parejas, sobre todo cuando se toma en cuenta que una de las metas en los manejos de fertilidad es la búsqueda de euploidia, de tal forma que en un modelo clínico predictivo utilizado para estimar el número de ovocitos maduros (metafase II) necesarios para obtener al menos un embrión euploide en parejas sometidas a técnicas de reproducción asistida indica que en 13 (IC 95\%: 11-16), 16 (IC 95\%: 13-20) y 19 (IC 95\%: 15-25) se necesitan ovocitos para las mujeres de 38, 39 y 40 años, respectivamente. Por el contrario, sólo en cinco (IC 95\%: 4-6) y seis (IC 95\%: 5-7) se requieren ovocitos para pacientes de 33 y 34 años que tengan al menos un embrión euploide. ${ }^{8}$

Desde la reciente aparición del virus hasta la fecha se desconocen muchas partes del comportamiento de este agente, incluida su epidemiología, el comportamiento entre razas, y algunas comorbilidades como es la infertilidad. No se tienen reportes de incidencia y prevalencia de la infección por COVID-19 en la población mexicana con problemas de fertilidad, ${ }^{9}$ por lo que el objetivo del estudio fue describir la incidencia de infección por virus de SARSCoV-2 en centros de fertilidad en la Ciudad de México.

\section{MATERIAL Y MÉTODOS}

Es un estudio descriptivo, transversal, observacional, retrospectivo, multicéntrico llevado a cabo en tres clínicas de reproducción asistida de la Ciudad de México de marzo a diciembre de 2020. Se realizó una revisión de los expedientes clínicos y la información recabada se añadió al programa Excel y posteriormente al programa SPSS v2. Se incluyeron mujeres ovodonadoras y mujeres candidatas a procedimientos de reproducción asistida, las cuales se realizaron prueba de proteína $C$ reactiva (PCR) para SARS-CoV-2 previo a su procedimiento; se excluyó a aquéllas con expediente incompleto. Se realizó estadística descriptiva obteniendo frecuencias, media y desviación estándar, y bajo pruebas de normalidad se realizó estadística inferencial corroborando la significancia estadística. Estudio aprobado para su realización por parte del comité de ética de la institución. 


\section{RESULTADOS}

Se obtuvo una muestra de 50 pacientes de tres centros de fertilidad en la Ciudad de México, de las cuales 42 eran infértiles y ocho ovodonadoras, con una media de edad de $35.4 \pm 6.25$ años, peso $61.5 \pm 8.37 \mathrm{~kg}$, talla $1.60 \pm$ $0.057 \mathrm{~m}$, índice de masa corporal (IMC) $23.8 \pm 3.64$. Se encontró 59.5\% de las pacientes con infertilidad primaria y $40.5 \%$ con infertilidad secundaria, sólo $23 \%$ presentaba comorbilidades, predominando en $10 \%$ hipotiroidismo y $8 \%$ con ingesta de levotiroxina con predominio de esta patología como factor endocrinológico de infertilidad. Alrededor de $55 \%$ de las pacientes candidatas a procedimiento de reproducción asistida tenían 37 años en adelante (Tabla 1).

Se obtuvieron cuatro pacientes con PCR positiva para SARS-CoV-2 $(p=0.041)$, tres de ellas habían estado en contacto con personas positivas, y una con síntomas respiratorios en los últimos 15 días $(\mathrm{p}=0.371)$ y en contacto con personas con síntomas respiratorios $(p=0.035)$, sólo $6 \%$ eran personal de salud ( $p=0.041$ ), $8 \%$ en contacto con una persona positiva en casa $(p=0.003$ ) (Tabla 2). Dentro de los procedimientos realizados se presentaron: transferencia, punción e histeroscopia en las pacientes, postergando a las pacientes positivas hasta contar con PCR para SARS-CoV-2 negativo y buscando otro tipo de anestesia en la que no exista aerolización. Siempre procurando las máximas medidas de protección sanitaria en las pacientes $y$ en el personal interno de los centros de fertilidad.

\section{DISCUSIÓN}

En el inicio de esta pandemia se recomendó postergar el embarazo debido a que es lo que se había manejado con las pandemias previas como la del virus de la inmunodeficiencia humana $(\mathrm{VIH})$, la de la influenza $\mathrm{N} 1 \mathrm{H} 1$ y la del virus del Zika, pandemias donde la experiencia nos demostró que ante el ataque en la población de un nuevo agente, es necesario primero conocer la enfermedad, entenderla lo mejor posible y tener evidencia de cómo puede modificar la morbilidad de la paciente y sus efectos en el producto. ${ }^{10}$

Actualmente se sabe que las pacientes embarazadas enfermas de COVID-19 tienen 1.5 veces más riesgo de ser admitidas en una unidad de cuidados intensivos, y 1.7 veces más riesgo de ser intubadas cuando se comparan contra mujeres no embarazadas; sin embargo, la mortalidad no se ve modificada entre estos dos grupos por la infección. Aunque se ha evidenciado la transmisión vertical de SARSCoV-2, no se ha demostrado que pueda tener un efecto teratogénico en el producto, pero sí se ha observado la transmisión de anticuerpos de forma benéfica. ${ }^{11}$
Aunque no se ha demostrado que el COVID-19 pueda afectar directamente la fertilidad de las mujeres de forma directa, hay evidencia de que el estrés psicológico ante el brote de este patógeno ha Ilegado. En un artículo original se describieron las actitudes, percepciones y reacciones psicológicas de pacientes infértiles, en las cuales se suspendió el tratamiento de fertilidad; en algunos lugares del mundo se cerraron varios centros de

Tabla 1: Factores sociodemográficos $(\mathrm{N}=50)$.

Edad (años)*
Peso $(\mathrm{kg})^{\star}$
Talla $(\mathrm{m})^{\star}$
Índice masa corporal*
Comorbilidades, n (\%)
Asma
Deficiencia de proteína S
Hipotiroidismo
VIH
X frágil
Obesidad
Negados
Medicamentos, $\mathrm{n}(\%)$
Levotiroxina
Negados

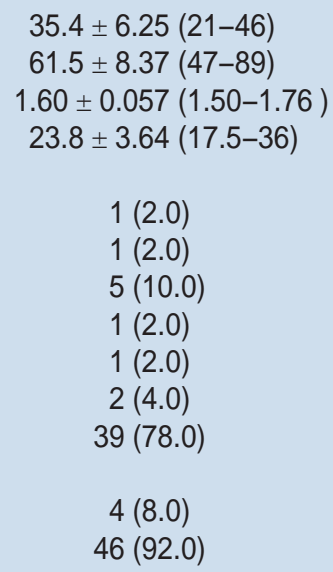

\begin{tabular}{ll}
\hline Primaria & Secundaria \\
$(\mathrm{N}=25)$ & $(\mathrm{N}=17)$
\end{tabular}

$\begin{array}{lcc}\begin{array}{l}\text { Factor endocrinológico } \\ \text { Hiperprolactinemia }\end{array} & 1(4.0) & 0(0) \\ \text { Hipotiroidismo } & 1(4.0) & 1(5.9) \\ \text { X frágil } & 1(4.0) & 0(0) \\ \text { Negados } & 22(88.0) & 16(94.1) \\ \text { Factor tubárico } & & \\ \text { Endometriosis } & 0(0) & 1(5.9) \\ \text { OTB } & 0(0) & 1(5.9) \\ \text { Negados } & 25(100.0) & 15(88.2) \\ \text { Factor uterino } & & \\ \text { Mioma } & 0(0) & 1(5.9) \\ \text { Negados } & 25(100.0) & 16(94.1) \\ \text { Factor masculino } & & \\ \text { Enterococo en semen } & 1(4.0) & 1(5.9) \\ \text { Teratozoospermia } & 1(4.0) & 1(5.9) \\ \text { Astenozoospermia } & 0(0) & 1(5.9) \\ \text { Negados } & 23(92.0) & 14(82.4)\end{array}$

* Datos expresados en: media \pm desviación estándar (rango intercuartílico). $\mathrm{VIH}=$ virus de inmunodeficiencia humana; OTB = obstrucción tubaria bilateral. 
Tabla 2: Relación de contacto con la infección SARS-CoV-2 previo a la realización del procedimiento.

\begin{tabular}{|c|c|c|c|c|}
\hline & \multicolumn{2}{|c|}{ Infertilidad, n (\%) } & \multirow[b]{2}{*}{$\begin{array}{l}\text { Ovodonadoras } \\
\qquad(\mathrm{N}=8)\end{array}$} & \multirow[b]{2}{*}{$\mathrm{p}$} \\
\hline & $\begin{array}{l}\text { Primaria } \\
(\mathrm{N}=25)\end{array}$ & $\begin{array}{l}\text { Secundaria } \\
\qquad(N=17)\end{array}$ & & \\
\hline Resultado PCR & & & & 0.041 \\
\hline Positivo & $2(8.0)$ & $0(0)$ & $2(25.0)$ & \\
\hline Negativo & $23(92.0)$ & $17(100.0)$ & $6(75.0)$ & \\
\hline Síntomas respiratorios en los últimos 15 días & & & & 0.371 \\
\hline Sí & $0(0)$ & $1(5.9)$ & $0(0)$ & \\
\hline No & $25(100.0)$ & $16(94.1)$ & $8(100.0)$ & \\
\hline Contacto con personas con síntomas respiratorios & & & & 0.035 \\
\hline Sí & $0(0)$ & $1(5.9)$ & $2(25.0)$ & \\
\hline No & $25(100.0)$ & $16(94.1)$ & $6(75.0)$ & \\
\hline Personal de salud & & & & 0.041 \\
\hline Sí & $1(4.0)$ & $0(0)$ & $2(25.0)$ & \\
\hline No & $24(96.0)$ & $17(100.0)$ & $6(75.0)$ & \\
\hline Contacto con persona positiva & & & & 0.003 \\
\hline Sí & $0(0)$ & $1(5.9)$ & $3(37.5)$ & \\
\hline No & $25(100.0)$ & $16(94.1)$ & $5(62.5)$ & \\
\hline
\end{tabular}

infertilidad, como consecuencia, se suspendieron los tratamientos de las pacientes que estaban en protocolos de fertilización in vitro (FIV). El hecho de verse obligadas a cancelar sus tratamientos incrementó significativamente el estrés y la depresión de estas pacientes, quienes perciben esta situación como un evento incontrolable y muy estresante, $38.9 \%$ de las pacientes presentaron angustia durante la suspensión del tratamiento. Ninguna de las características de fondo (edad, estado civil, paridad, nivel económico o duración de los tratamientos) tuvo una contribución significativa. Sentirse impotente después de la suspensión de los tratamientos se asoció con mayor angustia $(p<0.01)$. Mayor autodominio y apoyo social percibido se asociaron con angustia $(p<$ 0.01). A pesar de las recomendaciones de los sectores de salud, $72 \%$ de las pacientes deseaba reanudar el tratamiento en el momento de la encuesta. ${ }^{12}$

Contamos con una edad media en las pacientes infértiles de 35 años, lo que provocó sufrimiento psicológico cuando se cerraron los centros de infertilidad, tal como lo demostró Bovin en su estudio, el cual evidenció que las pacientes infértiles consideran el cierre de las clínicas donde llevaban sus tratamientos como un evento estresante debido a la incertidumbre y sensación de amenaza a su deseo de maternidad. Las pacientes evaluaron el cierre de la clínica de fertilidad como un impacto más negativo que positivo en sus vidas y como muy o extremadamente incontrolable y estresante $(p<0.001) .^{13}$

Se observa en este estudio que la incidencia de presentar infección por COVID-19 dentro del centro de reproducción asistida es muy baja. Hasta ahora se ha evidenciado el efecto deletéreo que puede tener en las pacientes el postergar tratamientos de fertilidad debido al COVID-19, por lo que suspender ciclos o cerrar los centros de infertilidad no es la solución. La solución es crear estrategias y recomendaciones para que las pacientes puedan seguir con sus proyectos de maternidad. ${ }^{14}$

Las pacientes ovodonadoras, aunque son un número menor en nuestra muestra, suelen ser mujeres trabajadoras que por ser de menor edad no pueden cumplir con las medidas de aislamiento en comparación con las pacientes infértiles. Hasta donde alcanza nuestra búsqueda, no hay estudios previos que refieran las tasas de incidencia que tiene este agente viral en las pacientes del centro de fertilidad, por lo que nuestro estudio podría considerarse un primer paso para que las pacientes infértiles puedan continuar, de una manera segura, sus tratamientos de fertilidad.

\section{CONCLUSIONES}

La incidencia de infección por SARS-CoV-2 en los centros de fertilidad es significativamente baja con las medidas adecuadas para la realización de los procedimientos, 
cuidando la salud de las pacientes de manera integral y del personal. En estas pacientes es preferible no retrasar los procedimientos por el deseo de concepción y la edad materna avanzada que presentan la mayoría.

Es recomendable realizar una prueba RT-PCR para SARS-CoV-2 a todas las pacientes que van a ingresar a un quirófano de infertilidad, pues el hecho de que la paciente salga positiva, permite planear el tipo de anestesia que sea libre de aerosoles; ya que se puede decidir cancelar, o en lugar de usar anestesia general con el equipo de protección adecuado se puede realizar el procedimiento con anestesia regional. La enfermedad por COVID-19 tiene mucho trasfondo aún por investigar. Al continuar el estudio en un futuro, el resultado reforzará nuestras conclusiones.

\section{REFERENCIAS}

1. Vaiarelli A, Bulletti C, Cimadomo D, Borini A, Alviggi C, Ajossa S et al. COVID-19 and ART: the view of the Italian Society of Fertility and Sterility and Reproductive Medicine. Reprod Biomed Online [Internet]. 2020; 40 (6): 755-759. Available in: http://dx.doi. org/10.1016/j.rbmo.2020.04.003

2. Li Q, Guan X, Wu P, Wang X, Zhou L, Tong Y et al. Early transmission dynamics in wuhan, china, of novel coronavirus-infected pneumonia. N Engl J Med [Internet]. 2020; 382 (13): 1199-1207. Available in: http://dx.doi.org/10.1056/NEJMoa2001316

3. Lauer SA, Grantz KH, Bi Q, Jones FK, Zheng Q, Meredith HR et al. The incubation period of coronavirus disease 2019 (COVID-19) from publicly reported confirmed cases: estimation and application. Ann Intern Med [Internet]. 2020; 172 (9): 577-582. Available in: http:// dx.doi.org/10.7326/M20-0504

4. COVID-19 UNAM-Mexico [Internet]. [Cited June 19, 2020]. Available in: https://covid19.ciga.unam.mx/

5. Albertini DF. Buffering reproductive science in the era of COVID-19. J Assist Reprod Genet [Internet]. 2020; 37 (5): 1017. Available in: http://dx.doi.org/10.1007/s10815-020-01824-4

6. Fan C, Lei D, Fang C, Li C, Wang M, Liu Y et al. Perinatal Transmission of 2019 Coronavirus Disease-Associated Severe Acute Respiratory Syndrome Coronavirus 2: Should We Worry? Clinical Infectious Diseases. 2020; 72 (5): 862-864.
7. Alviggi C, Esteves SC, Orvieto R, Conforti A, La Marca A, Fischer $\mathrm{R}$ et al. COVID-19 and assisted reproductive technology services: repercussions for patients and proposal for individualized clinical management. Reprod Biol Endocrinol [Internet]. 2020; 18 (1): 45. Available in: http://dx.doi.org/10.1186/s12958-020-00605-z

8. Esteves SC, Carvalho JF, Bento FC, Santos J. A novel predictive model to estimate the number of mature oocytes required for obtaining at least one euploid blastocyst for transfer in couples undergoing in vitro fertilization/intracytoplasmic sperm injection: the ART calculator. Front Endocrinol (Lausanne) [Internet]. 2019; 10: 99. Available in: http://dx.doi.org/10.3389/fendo.2019.00099

9. Poseidon Group (Patient-Oriented Strategies Encompassing IndividualizeD Oocyte Number), Alviggi C, Andersen CY, Buehler K, Conforti A, De Placido G et al. A new more detailed stratification of low responders to ovarian stimulation: from a poor ovarian response to a low prognosis concept. Fertil Steril [Internet]. 2016; 105 (6): 1452-1453. Available in: http://dx.doi.org/10.1016/j. fertnstert.2016.02.005

10. Diccionario de Cáncer del NCl [Internet]. 2011 [citado el 26 de octubre de 2020]. Disponible en: https://www.cancer.gov/espanol/ publicaciones/diccionario

11. Rasmussen SA, Lyerly AD, Jamieson DJ. Delaying pregnancy during a public health crisis - examining public health recommendations for Covid-19 and beyond. N Engl J Med [Internet]. 2020; 383 (22): 2097-2099. Available in: http://dx.doi.org/10.1056/NEJMp2027940

12. Ben-Kimhy R, Youngster M, Medina-Artom TR, Avraham S, Gat I, Haham LM et al. Fertility patients under COVID-19: attitudes, perceptions, and psychological reactions. Hum Reprod [Internet]. 2020; 35 (12): 2774-2783. Available in: http://dx.doi.org/10.1093/ humrep/deaa248

13. Boivin J, Harrison C, Mathur R, Burns G, Pericleous-Smith A, Gameiro S. Patient experiences of fertility clinic closure during the COVID-19 pandemic: appraisals, coping and emotions. Hum Reprod [Internet]. 2020; 35 (11): 2556-2566. Available in: http://dx.doi.org/10.1093/ humrep/deaa218

14. Mo Y, Wan R, Zhang Q. Application of reverse transcription-PCR and real-time PCR in nanotoxicity research. Methods Mol Biol [Internet]. 2012; 926: 99-112. Available in: http://dx.doi.org/10.1007/978-162703-002-1_7

Conflicto de intereses: No hubo conflicto de intereses al realizar este estudio. 